\title{
Erratum
}

Jonathan Hennessy*, Tirthankar Dasgupta, Luke Miratrix, Cassandra Pattanayak, and Pradipta Sarkar

\section{Erratum to: A Conditional Randomization Test for Covariate Imbalance}

https://doi.org/10.1515/jci-2017-0023

Erratum to: Hennessy J, Dasgupta T, Miratrix L, Pattanayak C, Sarkar P. A conditional randomization test to account for covariate imbalance in randomized experiments. J Causal Inference 2016;4(1):61-80 (https://doi.org/10.1515/jci-2015-0018).

There was an error in Hennessy et al. [1] and we are very grateful to Peng Ding for pointing it out.

Proposition 1, restated below, is incorrect, our proof being a mis-application of a result from Rosenbaum [2].

Proposition 1. Let $X$ denote a categorical covariate with $J$ levels, observed after a two-armed randomized experiment is conducted with $N$ units. Let $N_{j}$ denote the observed number of units that belong to stratum $j$, and let $N_{T j}$ and $N_{C j}$ denote the number of units assigned to treatment and control respectively, in stratum $j$, such that $N_{T j}+N_{C j}=N_{j}$, and $\sum_{j=1}^{J} N_{j}=N$. Then the conditional randomization test using the simple difference test statistic $\hat{\tau}_{s d}=\bar{Y}_{T}^{\text {obs }}-\bar{Y}_{C}^{\text {obs }}$ and the balance function $\left(N_{T 1}, \ldots, N_{T J}\right)$ is equivalent to the conditional randomization test using the composite test statistic $\hat{\tau}_{p s}=\sum_{j=1}^{J} \frac{N_{j}}{N} \hat{\tau}_{s d, j}$, where $\hat{\tau}_{s d, j}$ denotes the simple difference test statistic for the jth stratum.

We can show the proposition is not true by a simple counterexample. In order for the two conditional tests to be equivalent, they must yield the same p-values. Consider the situation where $N=5, X=(1,1,1,2,2)$, $\mathbf{w}=(1,0,0,1,0)$, and $\mathbf{y}^{\text {obs }}=(1.13,0.49,-0.31,0.98,1.68)$. In this case, $\hat{\tau}_{s d}=0.435$ and $\hat{\tau}_{p s}=0.344$. To find the p-values for the conditional test, we consider the values of the test statistics across all 6 alternative randomizations where $N_{T 1}=1$ and $N_{T 2}=1$.

To calculate the $\mathrm{p}$-values, we find the proportion of test statistics as or more extreme than the observed. For $\hat{\tau}_{s d}=0.435$, there are three test statistics as large or larger $(0.435,0.485,1.018)$, so the 2-sided $p$-value is $2 \cdot 3 / 6=1$. For $\hat{\tau}_{p s}=0.344$, there are two test statistics as large or larger $(0.344,0.904)$, so the 2 -sided $p$-value is $2 \cdot 2 / 6=2 / 3$. Since the $p$-values do not agree, the tests are not equivalent.

The incorrect proof in Appendix A mis-applied a result from Rosenbaum [2] that showed that in a linear regression of the response on the treatment indicator and covariates, a conditional randomization test based on the treatment indicator coefficient is equivalent to the conditional randomization test based on the simple difference test statistic. This proof rests on the fact that the columns corresponding to the covariates are fixed across randomizations. While our $\hat{\tau}_{p s}$ does equal a regression coefficient, that regression includes interactions between the treatment indicator and the covariates. However, these interaction terms are not

*Corresponding author: Jonathan Hennessy, Department of Statistics, Harvard University, Cambridge, MA, USA, E-mail: jonathan.hennessy@gmail.com

Tirthankar Dasgupta, Department of Statistics, Harvard University, Cambridge, MA, USA, E-mail: dasgupta@stat.harvard.edu Luke Miratrix, Graduate School of Education, Harvard University, Cambridge, MA, USA, E-mail: Imiratrix@g.harvard.edu Cassandra Pattanayak, Quantitative Analysis Institute, Wellesley College, Wellesley, MA, USA, E-mail: cpattanayak@wellesley.edu

Pradipta Sarkar, Principal Scientist, Procter \& Gamble International Operations, Singapore, Singapore,

E-mail: sarkar.p@pg.com 
Table 1: Alternative randomizations: For each alternative randomization where $N_{T 1}=1$ and $N_{T 2}=1$, we calculate both test statistics.

\begin{tabular}{lrr}
\hline Randomization & $\hat{\boldsymbol{\tau}}_{\text {sd }}$ & $\hat{\boldsymbol{\tau}}_{\text {ps }}$ \\
\hline$(1,0,0,1,0)$ & 0.435 & 0.344 \\
$(0,1,0,1,0)$ & -0.098 & -0.232 \\
$(0,0,1,1,0)$ & -0.765 & -0.952 \\
$(1,0,0,0,1)$ & 1.018 & 0.904 \\
$(0,1,0,0,1)$ & 0.485 & 0.328 \\
$(0,0,1,0,1)$ & -0.182 & -0.392 \\
\hline
\end{tabular}

fixed across the different randomizations and we ignored this fact in the proof. In the proof in Appendix A, we incorrectly assumed $k_{1}=\mathbf{w}^{T} \mathbf{F}\left(\mathbf{F}^{T} \mathbf{F}\right)^{-1} \mathbf{F}^{T} \mathbf{y}^{\text {obs }}$ is a constant. While $\mathbf{w}^{T} \mathbf{F}\left(\mathbf{F}^{T} \mathbf{F}\right)^{-1}$ is a constant, $\mathbf{F}^{T} \mathbf{y}^{\text {obs }}$ is not. ${ }^{1}$

Note that the main results and conclusions regarding conditional randomization tests from Hennessy et al. [1] do not depend on the proposition. The proposition was only used in the simulation study to reduce the number of tests to be compared. Rather than reporting the conditional tests using both $\hat{\tau}_{s d}$ and $\hat{\tau}_{p s}$, we only reported results using $\hat{\tau}_{s d}$. However, in the specific simulation setting we explored, $\hat{\tau}_{p s}$ is, in fact, a monotonic function of $\hat{\tau}_{s d}$ when conditioning on the observed balance because there are two strata of equal size and the treated and control groups are of equal size. In this situation, the conditional tests using $\hat{\tau}_{s d}$ and $\hat{\tau}_{p s}$ are equivalent. We verify this fact below. For this situation, our test statistics can be expanded as

$$
\begin{aligned}
\hat{\tau}_{s d} & =\left(\frac{N_{T 1}}{N_{T}} \bar{Y}_{T 1}^{\mathrm{obs}}+\frac{N_{T 2}}{N_{T}} \bar{Y}_{T 2}^{\mathrm{obs}}\right)-\left(\frac{N_{C 1}}{N_{C}} \bar{Y}_{C 1}^{\mathrm{obs}}+\frac{N_{C 2}}{N_{C}} \bar{Y}_{C 2}^{\mathrm{obs}}\right) \\
& =\frac{2}{N}\left(N_{T 1} \bar{Y}_{T 1}^{\mathrm{obs}}+N_{T 2} \bar{Y}_{T 2}^{\mathrm{obs}}-N_{C 1} \bar{Y}_{C 1}^{\mathrm{obs}}-N_{C 2} \bar{Y}_{C 2}^{\mathrm{obs}}\right)
\end{aligned}
$$

and

$$
\begin{aligned}
\hat{\tau}_{p s} & =\frac{N_{1}}{N}\left(\bar{Y}_{T 1}^{\mathrm{obs}}-\bar{Y}_{C 1}^{\mathrm{obs}}\right)+\frac{N_{2}}{N}\left(\bar{Y}_{T 2}^{\mathrm{obs}}-\bar{Y}_{C 2}^{\mathrm{obs}}\right) \\
& =\frac{1}{2}\left(\bar{Y}_{T 1}^{\mathrm{obs}}+\bar{Y}_{T 2}^{\mathrm{obs}}-\bar{Y}_{C 1}^{\mathrm{obs}}-\bar{Y}_{C 2}^{\mathrm{obs}}\right) .
\end{aligned}
$$

We then show that $\hat{\tau}_{p s}$ is a monotonic function of $\hat{\tau}_{s d}$ by showing that

$$
\hat{\tau}_{p s}=\frac{N}{4 N_{T 1} N_{C 1}}\left(\frac{N}{4}\left(\hat{\tau}_{s d}+2 \bar{Y}^{\mathrm{obs}}\right)-N_{T 1} \bar{Y}_{1}^{\mathrm{obs}}-N_{T 2} \bar{Y}_{2}^{\mathrm{obs}}\right) .
$$

The proof is available upon request.

If we were to include the conditional randomization test using $\hat{\tau}_{p s}$ in the simulation study, the results would be the same as those reported for the conditional randomization test using $\hat{\tau}_{s d}$. We leave a formal comparison of conditional randomization tests using $\hat{\tau}_{s d}$ and $\hat{\tau}_{p s}$ for future work.

\section{References}

1. Hennessy J, Dasgupta T, Miratrix L, Pattanayak C, Sarkar P. A conditional randomization test to account for covariate imbalance in randomized experiments. J Causal Inference 2016;4(1):61-80.

2. Rosenbaum PR. Conditional permutation tests and the propensity score in observational studies. J Am Stat Assoc 1984;79(387):565-74.

1 We also incorrectly defined the covariate dummies and interaction terms in Appendix A. The covariate dummies should follow the standard treatment contrast coding. So with $J$ strata, use dummy variables $Z_{i 2}, \ldots Z_{i J}$ to encode the strata for unit $i$, giving $J-1$ interaction terms of the form $W_{i} \cdot\left(Z_{i 2}-\bar{Z}_{2}\right), \ldots, W_{i} \cdot\left(Z_{i J}-\bar{Z}_{J}\right)$, where $\bar{Z}_{j}=\frac{1}{N} \sum Z_{i j}$. 